\title{
Renal Function During an Open-Label Prospective Observational Trial of Sitagliptin in Patients With Diabetes: A Sub-Analysis of the JAMP Study
}

\author{
Osamu Tomonaga ${ }^{\mathrm{a}, 1}$, Hiroshi Sakura ${ }^{\mathrm{b}}$, Naotake Hashimoto ${ }^{\mathrm{c}}$, Kazuo Sasamoto ${ }^{\mathrm{d}}$, Hiroshi Ohashie, \\ Sumiko Hasumif ${ }^{\mathrm{f}}$, Noriko Ujihara ${ }^{\mathrm{g}}$, Tadasu Kasahara ${ }^{\mathrm{h}}$, Hideo Nunome ${ }^{\mathrm{i}}$, Masashi Honda ${ }^{\mathrm{j}}$, \\ Yasuhiko Iwamoto ${ }^{\mathrm{k}}$, for the JAMP Study Investigators
}

\begin{abstract}
Background: The aim of the study was to determine the effects of sitagliptin on renal function in a diabetic population including patients with normal renal function.

Methods: We analyzed the association between 12-month, $50 \mathrm{mg} /$ day sitagliptin and renal function in outpatients with type 2 diabetes mellitus and poor blood glucose control in a subset of patients in the larger Januvia Multicenter Prospective Trial in Type 2 Diabetes observational study. Stratified analyses of changes in estimated glomerular filtration rate (eGFR) and urinary albumin-to-creatinine ratio (UACR) were performed. Factors associated with changes in eGFR at 3 months were examined by multivariate regression analysis.
\end{abstract}

Manuscript submitted October 10, 2017, accepted October 23, 2017

${ }^{a}$ Diabetes and Lifestyle Center, Tomonaga Clinic, Shinyon Curumu Building 9F, 4-2-23, Shinjuku, Shinjuku-ku, Tokyo 160-0022, Japan

${ }^{b}$ Department of Medicine, Tokyo Women's Medical University, Medical Center East, 2-1-10, Nishiogu, Arakawa-ku, Tokyo 116-8567, Japan

'Department of Diabetes, Endocrine and Metabolic Diseases, Tokyo Women's Medical University Yachiyo Medical Center, 477-96, Owadashinden, Yachiyo-shi, Chiba 276-0046, Japan

IInternal Medicine, Suzuki Clinic, 2-10-14, Koyasu-machi, Hachioji-shi, Tokyo 192-0904, Japan

'Internal Medicine, Oyama East Clinic, 1-32-1, Ekihigashi-dori, Oyama-shi, Tochigi 323-0022, Japan

Internal Medicine, Nishiyamado-Keiwa Hospital, 3247-1, Kounosu, Nakashi, Ibaraki 311-0133, Japan

gDepartment of Medicine, Diabetes Center, Institute of Geriatrics, Tokyo Women's Medical University, Shibuya Cross Tower 22F, 2-15-1, Shibuya, Shibuya-ku, Tokyo 150-0002, Japan

hJosai Hospital, 2-42-11, Kamiogi, Suginami-ku, Tokyo 167-0043, Japan iDiabetes Center, Edogawa Hospital, Medical Plaza Shinozaki, SK Building, Shinozakimachi, Edogawa-ku, Tokyo 133-0057, Japan

${ }^{j}$ Nishikawa Clinic, 2-16-3, Towa, Adachi-ku, Tokyo 120-0003, Japan

kThe Institute for Adult Diseases, Asahi Life Foundation, Asahiseimeisunaga Building, 2-2-6, Nihonbashi Bakuro-cho, Chuo-ku, Tokyo 103-0002, Japan

${ }^{1}$ Corresponding Author: Osamu Tomonaga, Diabetes and lifestyle Center, Tomonaga Clinic, Shinyon curumu Building 9F, 4-2-23, Shinjuku, Shinjuku-ku, Tokyo 160-0022, Japan. Email: boss@tomonaga-clinic.com

doi: https://doi.org/10.14740/jocmr3225w
Results: Of the 779 patients enrolled, 585 were followed up for 12 months. eGFR decreased significantly from baseline at 3 and 12 months in patients with a baseline eGFR of $\geq 90 \mathrm{~mL} / \mathrm{min} / 1.73 \mathrm{~m}^{2}$ and in those with a baseline eGFR of $\geq 60$ to $<90 \mathrm{~mL} / \mathrm{min} / 1.73 \mathrm{~m}^{2}$. Conversely, eGFR tended to increase at 3 and 12 months in patients with a baseline eGFR of $\geq 45$ to $<60 \mathrm{~mL} / \mathrm{min} / 1.73 \mathrm{~m}^{2}$ and in those with a baseline eGFR of $\geq 30$ to $<45 \mathrm{~mL} / \mathrm{min} / 1.73 \mathrm{~m}^{2}$. UACR decreased significantly $(-21.6(-46.8,7.8))$ at 3 months in patients with a baseline UACR of $\geq 30 \mathrm{mg} / \mathrm{g}$ Cre. Multivariate regression analysis of factors associated with changes in eGFR at 3 months revealed that higher baseline eGFR and greater decline in UACR were associated with more conspicuous decreases in eGFR.

Conclusions: In this group of diabetic patients receiving sitagliptin, eGFR declined in patients with high baseline eGFR, but not in those with a low baseline eGFR.

Keywords: DPP-4 inhibitor; Prospective observational study; Renal function; Sitagliptin; JAMP

\section{Introduction}

Sitagliptin, a dipeptidyl peptidase-4 (DPP-4) inhibitor, improves blood glucose control by enhancing insulin secretion and inhibiting glucagon secretion in a blood glucose leveldependent manner via inhibition of GLP-1 inactivation and an action mediated by the DPP-4 substrate $[1,2]$. This agent permits a once-daily regimen, is associated with a low risk of hypoglycemia and weight gain [3], and lowers glycated hemoglobin (HbA1c) levels. Therefore, it has been widely used, not only as monotherapy but also as combination therapy with pioglitazone, metformin, voglibose, sulfonylureas, and other anti-diabetic agents [4-7].

Sitagliptin is renally excreted, hence dose adjustment should be considered in patients with diabetes mellitus and concomitant moderate or severe chronic kidney disease or end-stage renal disease requiring dialysis, in addition to considering the patient's tolerability to sitagliptin and the effects of sitagliptin on renal function $[8,9]$. However, renal impairment may be undetected in patients with diabetes. In a retro- 
spective database analysis, Meyers et al reported that patients with an estimated glomerular filtration rate (eGFR) of $<60$ $\mathrm{mL} / \mathrm{min} / 1.73 \mathrm{~m}^{2}$ accounted for $35.2 \%$ of 344,770 patients with type 2 diabetes mellitus (T2DM) [10]. Moreover, the Japanese Society for Dialysis Therapy reported that among 320,448 patients who were undergoing dialysis, $43.5 \%$ had diabetic nephropathy as primary disease [11], showing the need for appropriate treatment of diabetes that takes into consideration effects on renal function.

The effects of sitagliptin on renal function have been studied using eGFR and urinary albumin-to-creatinine ratio (UACR) as indices. In the study by Kawasaki et al sitagliptin was administered to patients with diabetic nephropathy and UACRs were decreased significantly after 3 months of sitagliptin therapy in patients with UACR $30-299$ or $\geq 300 \mathrm{mg} / \mathrm{g}$ Cre at baseline [12]. In addition, in a study of 4 years' administration of sitagliptin in elderly patients, eGFR tended to increase, although the difference from baseline was not significant [13]. Chan et al reported that in patients with a creatinine clearance of $<50 \mathrm{~mL} / \mathrm{min}$, UACR tended to decrease after 54 weeks of sitagliptin administration [14]. The aforementioned patients with reduced renal function generally showed a tendency for improvement; however, the results were not consistent when patients with normal renal function were considered. Mori et al reported a significant improvement in UACR after 6 months of sitagliptin therapy [15]. In contrast, in the Trial Evaluating Cardiovascular Outcomes with Sitagliptin (TECOS), eGFRs were significantly decreased 4 years after initiation of sitagliptin therapy, in the same manner as in those who received placebo [16]. In a Study of Safety and Efficacy of DPP-4 inhibitor in the Treatment of type 2 diabetes in Kanagawa (ASSET-K study), serum creatinine levels were increased significantly after 1 month of sitagliptin therapy [17].

The TECOS and ASSET-K studies are relatively largescale studies in which significant differences are more likely to be detected. These studies did not conduct a stratified analysis; thus, their results could not determine the effects of sitagliptin in patients with reduced renal function. In this regard, we thought it necessary to determine the actual effects of sitagliptin by conducting a stratified analysis of its effects on renal function in a population that includes diabetic patients with normal renal function.

In this study, we used follow-up data obtained 1 year after initiation of sitagliptin therapy from the JAMP study, a prospective observational study [18]. In this sub-analysis of the JAMP study, we performed an exploratory analysis involving stratification to examine the association between sitagliptin administration and changes in renal function.

\section{Materials and Methods}

\section{Study design}

This open-label, central registration, multicenter, prospective observational study was conducted at the Tokyo Women's Medical University Hospital and 69 collaborating institutions in Japan. Patients were enrolled from January 2011 to June
2013, and followed up until June 2014. This study was conducted in accordance with the study protocol, the Declaration of Helsinki, and the Ministry of Health, Labour and Welfare of Japan (MHLW) "Ethical Guidelines for Clinical Studies".

\section{Study subjects}

Study subjects were male or female, aged $\geq 20$ years, and outpatients with T2DM and poorly controlled blood glucose levels for at least 1 month despite diet/exercise therapy alone or in combination with anti-diabetic therapy. Before participation, all patients provided written informed consent.

The anti-diabetic drugs used were sitagliptin, low-dose glimepiride $(0.5$ - $1 \mathrm{mg})$, medium-dose glimepiride (1.5 - 2 $\mathrm{mg}$ ), biguanides, thiazolidines, $\alpha$-glucosidase inhibitors, or two or more of these drugs in combination. The criterion for inadequate blood glucose control was set at: $\mathrm{HbA} 1 \mathrm{c} \geq 6.9 \%$ or fasting blood glucose $130 \mathrm{mg} / \mathrm{dL}$ [19].

Patients who met any of the following criteria were excluded from the study: 1) history of severe ketosis, diabetic coma or pre-coma within the past 6 months; 2) severe infection before or after surgical treatment, or serious external injury; 3 ) pregnancy, possible pregnancy, or lactation; 4) moderate renal impairment (serum creatinine level $\geq 1.5 \mathrm{mg} / \mathrm{dL}$ in men and $\geq 1.3 \mathrm{mg} / \mathrm{dL}$ in women); 5) on insulin therapy; 6) receiving rapid-acting insulin secretagogues; 7) history of allergy to the ingredients of the study drug; and 8) a medical reason that makes the patient unsuitable for participation in the study as judged by the investigator.

\section{Methods}

The pre-existing therapy for type 2 diabetes was not changed during the observation period and entire study period (for the first 3 months after add-on sitagliptin). Thereafter, sitagliptin $(50 \mathrm{mg})$ was administered once daily as a first-line treatment (single-drug therapy) or as an additional treatment (combination therapy; Fig. 1). During the 3-month period following the initiation of sitagliptin treatment (baseline), administration of sitagliptin was continued without the addition of any other drugs or increase in dose. At 3 months, the sitagliptin dose was increased from 50 to $100 \mathrm{mg}$ /day and other antidiabetic drugs were added, changed or discontinued at the investigator's discretion. No restrictions were imposed on the use of drugs for treating concurrent diseases, but change in dose or the addition of new drugs during the study period was avoided whenever possible.

\section{Evaluation}

Any associations of sitagliptin with changes in renal function were evaluated through assessment of: 1) change in eGFR at 3 and 12 months from baseline; 2) changes in eGFR and HbA1c levels at 3 and 12 months from baseline in patients classified into four groups according to baseline eGFR: $\geq 30$ to $<45, \geq$ 


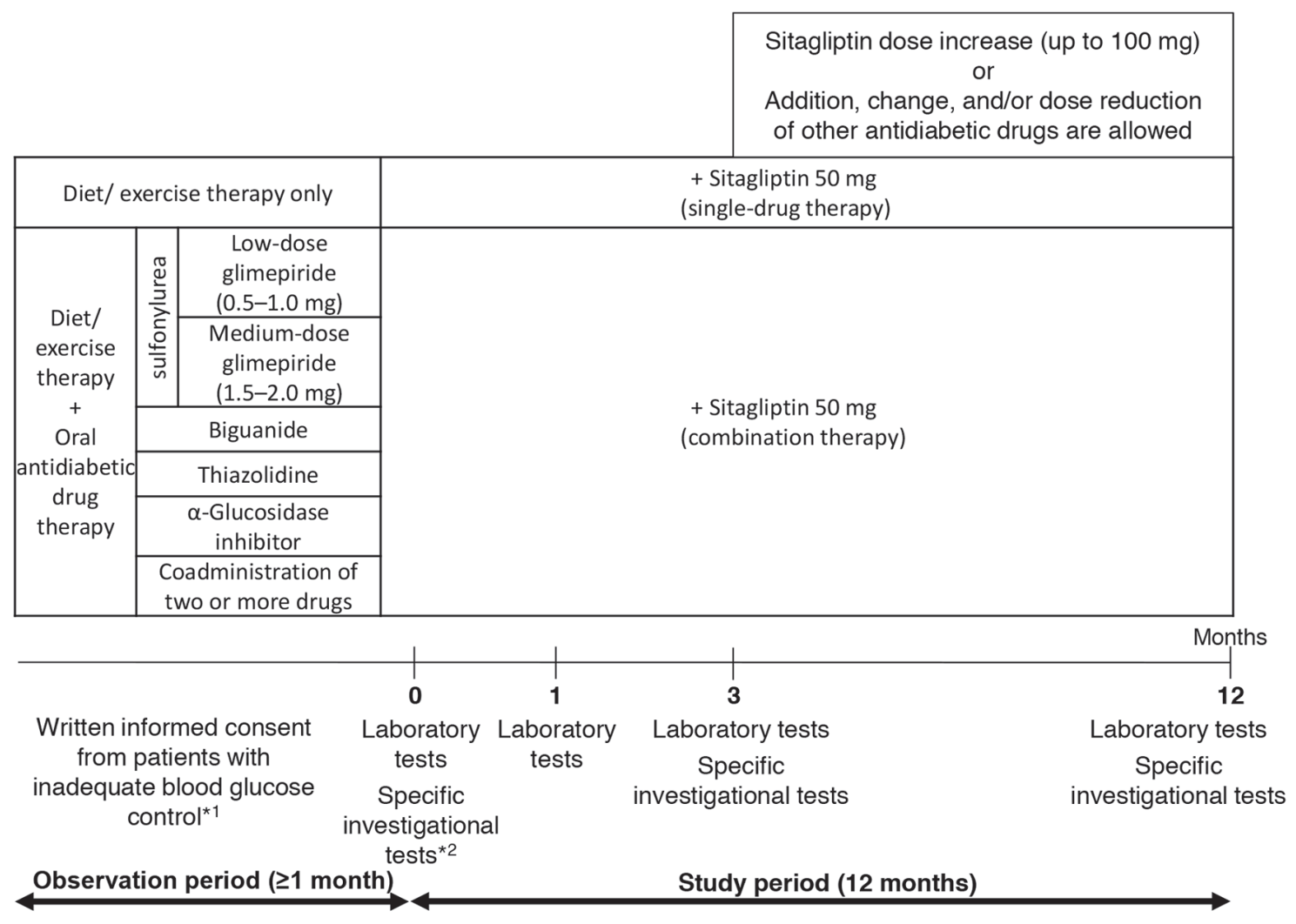

Figure 1. Study design. *1: criteria for inadequate blood glucose control: $\mathrm{HbA} 1 \mathrm{c}$ level $\geq 6.9 \%$ or fasting blood glucose level $\geq 130$ $\mathrm{mg} / \mathrm{dL}$. *2: specific investigational tests (optional): GA, 1.5AG, C-peptide, and proinsulin-to-insulin ratio.

45 to $<60, \geq 60$ to $<90$, or $\geq 90 \mathrm{~mL} / \mathrm{min} / 1.73 \mathrm{~m}^{2} ; 3$ ) change in UACR at 3 and 12 months from baseline in patients with a baseline UACR $\geq 30 \mathrm{mg} / \mathrm{g}$ Cre; and 4) multivariate analysis of factors that influenced change in eGFR at 3 months from baseline.

\section{Statistical analysis}

Changes in eGFR and HbA1c levels were evaluated using the paired $t$-test. Differences in changes in eGFR and HbA1c levels between the groups of patients stratified according to baseline eGFR were evaluated by ANOVA. Changes in UACRs were evaluated using the Wilcoxon signed-rank test. As for factors that might influence the change in eGFR at 3 months from baseline, we performed a single regression analysis of age, sex, duration of T2DM, use of antihypertensive drugs, treatment with angiotensin-converting enzyme inhibitors (ACEI) or angiotensin II receptor blockers (ARB), baseline HbA1c, eGFR, and systolic blood pressure (SBP), change in $\mathrm{HbA} 1 \mathrm{c}$, change in $\mathrm{SBP}$, and change in UACR. Subsequently, variables associated with changes in eGFRs in the single regression analysis were evaluated using multiple regression analysis.

The significance level was set at 5\% (two-sided). The JMP software program version 12.1.0 (SAS Institute Inc., Cary, NC, USA) was used for statistical analysis processing. The results of the analysis of UACRs are presented in the text as median (first quartile and third quartile). Other continuous variables are presented as mean \pm standard deviation (SD), and the nominal scale is the number $(\%)$ of patients. The units used for eGFR and UACR are $\mathrm{mL} / \mathrm{min} / 1.73 \mathrm{~m}^{2}$ and $\mathrm{mg} / \mathrm{g}$ Cre, respectively.

\section{Ethic approval and consent to participate}

All procedures followed were in accordance with the ethical standards of the responsible committee on human experimentation (institutional and national) and with the Helsinki Declaration of 1964 and later versions. The ethics committee at the Tokyo Women's Medical University approved the study (approval number: 2064) on January 11, 2011. Informed consent or substitute for it was obtained from all patients for being included in the study.

\section{Results}

Of the 779 patients with T2DM enrolled, 651 were included in the efficacy analysis (Fig. 2). Table 1 shows the demographic characteristics of the 651 patients who underwent efficacy evaluation in the JAMP study. The mean age of the patients was $63.8 \pm 11.8$ years, 434 were men $(66.7 \%)$, and mean disease duration was $8.8 \pm 6.7$ years.

In 491 patients who were followed up for 12 months, 


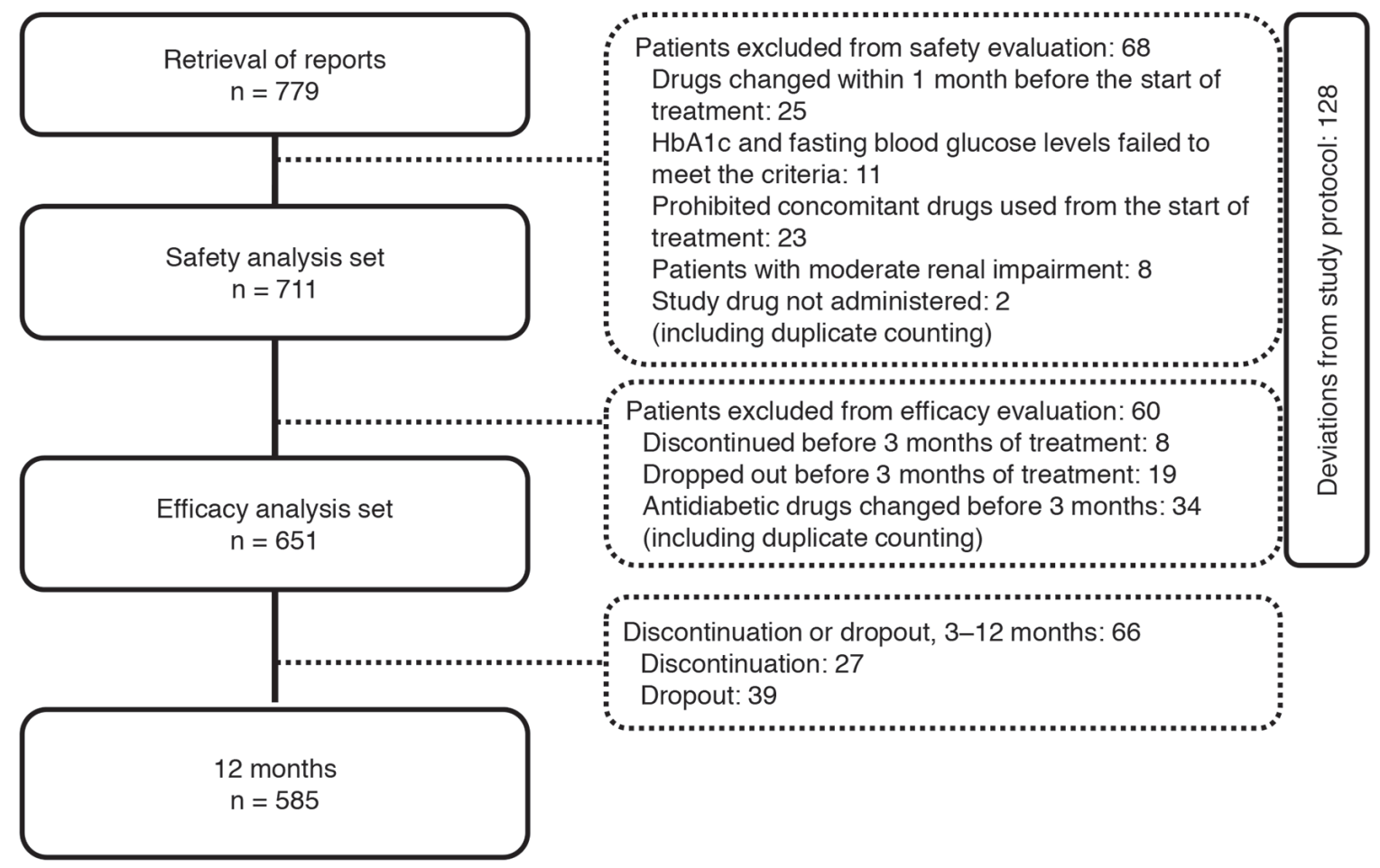

Figure 2. Participant flow in the study.

eGFR declined from $78.1 \pm 18.4$ at baseline to $75.4 \pm 18.1$ and $74.2 \pm 17.8$ at 3 and 12 months, respectively, both showing a significant decrease from the baseline value $(\mathrm{P}<0.05)$.
Figure 3 shows the changes in eGFR at 3 and 12 months in the four groups classified according to eGFR at baseline. A significant decrease in eGFR at 3 and 12 months from baseline

Table 1. Clinical Characteristics of Study Patients $(n=651)$

\begin{tabular}{llll}
\hline Parameter & Mean \pm SD or n (\%) & Parameter & Mean \pm SD or n (\%) \\
\hline Age (years) & $63.8 \pm 11.8$ & Arteriosclerosis obliterans & $55(8.4)$ \\
Sex (male \%) & $434(66.7)$ & Atrial fibrillation & $16(2.5)$ \\
BMI $\left(\mathrm{kg} / \mathrm{m}^{2}\right)$ & $25.2 \pm 4.2$ & Renal disease & $49(7.5)$ \\
Duration of T2DM (years) & $8.8 \pm 6.7$ & Hepatic disease & $56(8.6)$ \\
Abdominal circumference (cm) & $88.3 \pm 11.1$ & Myocardial infarction & $18(2.8)$ \\
HbA1c (\%) & $7.86 \pm 1.07$ & Cerebral stroke & $45(6.9)$ \\
Fasting blood glucose (mg/dL) & $159.2 \pm 41.5$ & Angina pectoris & $27(4.1)$ \\
HOMA-IR & $2.83 \pm 1.80$ & Cardiac failure & $11(1.7)$ \\
HOMA- $\beta(\%)$ & $32.1 \pm 27.1$ & Myocardial infarction & $18(2.8)$ \\
C-peptide (ng/mL) & $2.10 \pm 0.89$ & Diet/exercise therapy & $189(29)$ \\
SBP (mm Hg) & $130.9 \pm 14.9$ & Low-dose glimepiride $0.5-1.0 \mathrm{mg}$ & $72(11.1)$ \\
DBP (mm Hg) & $76.5 \pm 10.5$ & Medium-dose glimepiride $1.5-2.0 \mathrm{mg}$ & $50(7.7)$ \\
Hypertension & $393(60.4)$ & Biguanide & $99(15.2)$ \\
Dyslipidemia & $417(64.1)$ & Thiazolidine & $38(5.8)$ \\
Hyperuricemia & $65(10)$ & $\alpha-G I$ & $18(2.8)$ \\
Retinopathy & $48(7.4)$ & Multiple-drug co-administration & $185(28.4)$ \\
\hline
\end{tabular}

Data are represented as the mean \pm SD or $n$ (\%). BMI: body mass index; T2DM: type 2 diabetes mellitus; HbA1c: glycated hemoglobin; HOMA-IR: homeostatic model assessment for insulin resistance; HOMA- $\beta$ : homeostatic model assessment for $\beta$-cell function; SBP: systolic blood pressure; DBP: diastolic blood pressure; $\alpha-G I$ : $\alpha$-glucosidase inhibitor. 
a

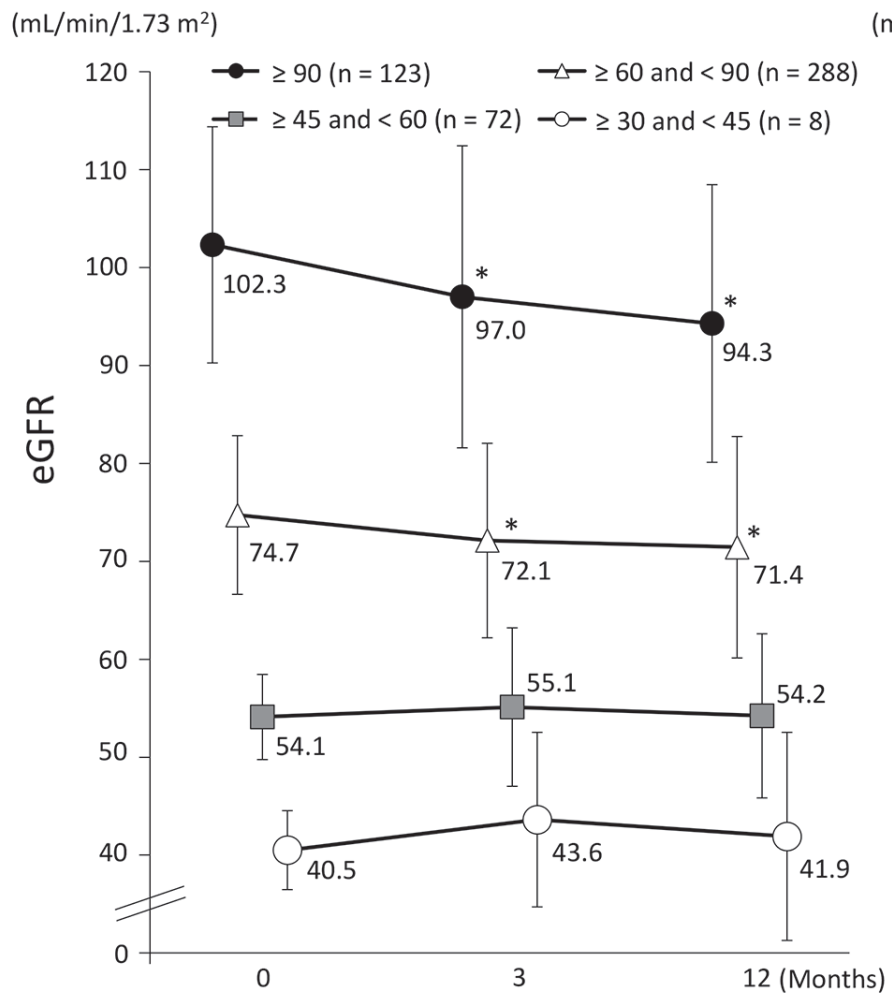

b

$\left(\mathrm{mL} / \mathrm{min} / 1.73 \mathrm{~m}^{2}\right)$

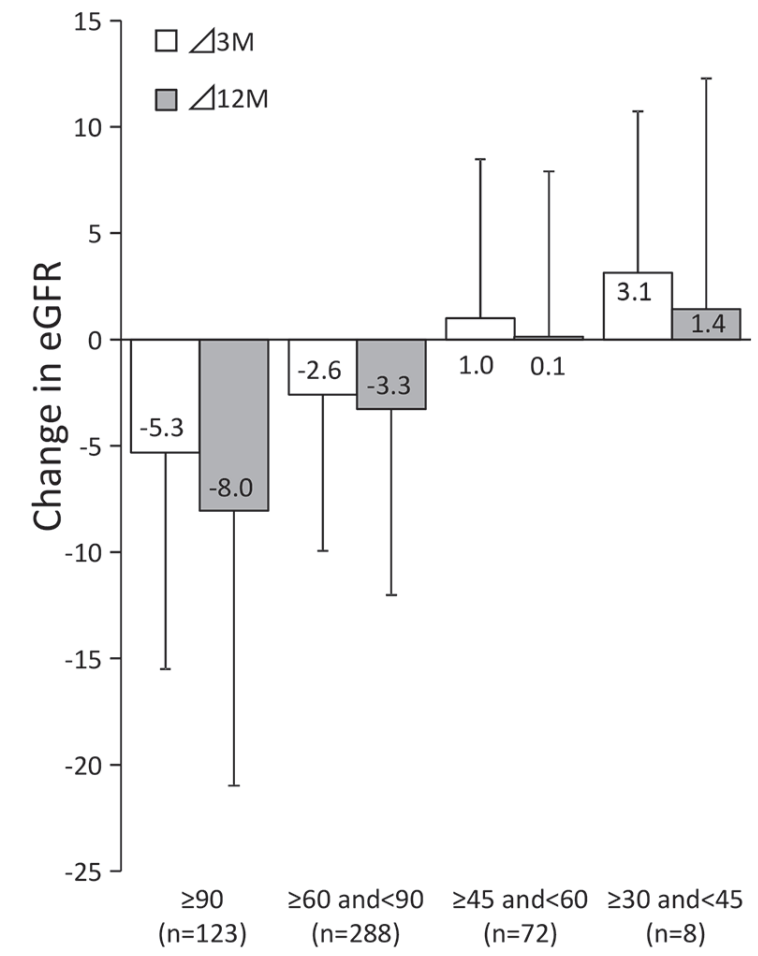

Figure 3. Changes in eGFR (categorized by baseline eGFR). \pm SD.

values was observed in patients with a baseline eGFR of $\geq 90$ and those with a baseline eGFR of $\geq 60$ to $<90$. On the other hand, patients with a baseline eGFR of $\geq 45$ to $<60$ or $\geq 30$ to $<45$ showed no significant difference from baseline but eGFR showed a tendency to increase in these groups at both 3 and 12 months. Regardless of the eGFR at baseline, HbA1c levels were decreased significantly at 3 and 12 months in all the patient groups (Fig. 4).

Median UACR (of patients with baseline UACR $\geq 30 \mathrm{mg} / \mathrm{g}$ Cre) ranged from $61.5(41.8,143.8)$ at baseline to $45.3(24.4$, $132.05)$ at 3 months and $52.1(20.5,130.35)$ at 12 months. Compared with baseline, UACR at 3 months was statistically significantly lower $(\mathrm{P}<0.05)$, but the difference at 12 months did not reach statistical significance (Fig. 5).

Table 2 shows the results of the evaluation of factors that may have influenced changes from baseline in eGFR at 3 months. Single regression analysis showed that eGFR at baseline, change in UACR, and change in HbA1c levels influenced the change in eGFR. Multiple regression analysis of these factors and two other factors with $\mathrm{P}$ values of $<0.2$, i.e., change in SBP and use of concomitant antihypertensive drugs, showed that the higher the eGFR at baseline and the greater the decline in UACR, the more conspicuous the decrease in eGFR.

\section{Discussion}

In patients with high eGFRs at baseline, eGFRs were decreased (a) ${ }^{*} \mathrm{P}<0.05$ vs. $0 \mathrm{M}$ paired $t$-test. (b) Values presented are mean

significantly after 3 months of sitagliptin therapy. In contrast, in patients with low eGFRs at baseline, eGFRs tended to increase after 3 months of sitagliptin therapy. Similar to previous large-scale clinical studies [16], eGFRs in the present study were significantly decreased in the overall patient population. However, we could not conclude that sitagliptin caused further deterioration of renal function in patients with reduced renal function.

In patients with UACRs of $\geq 30 \mathrm{mg} / \mathrm{g}$ Cre at baseline, UACRs were decreased significantly after 3 months of therapy, which suggests that renal function might have improved. Takihata et al also reported a significant improvement in UACR after 24 weeks of sitagliptin therapy, although a significant decline in eGFR was observed [20], supporting the results of the present study.

Multiple regression analysis revealed that the higher the eGFR at baseline and the greater the improvement in UACR at 3 months, the greater the decrease in eGFR. This finding suggests a correlation between decreased urinary protein levels and decreased eGFR.

The decreased eGFR may be explained by the following: improvement of osmotic diuresis in addition to improvement in blood glucose levels; elimination of hyperfiltration; and a direct effect of sitagliptin outside the pancreas. As the kidney has many GLP-1 receptors and expresses DPP-4, DPP-4 inhibitors are expected to exert a direct renoprotective effect. A study in hypertensive rats showed that $\mathrm{Na}$ excretion was facilitated by inhibition of NHE3 activity by sitagliptin [21]. 
a

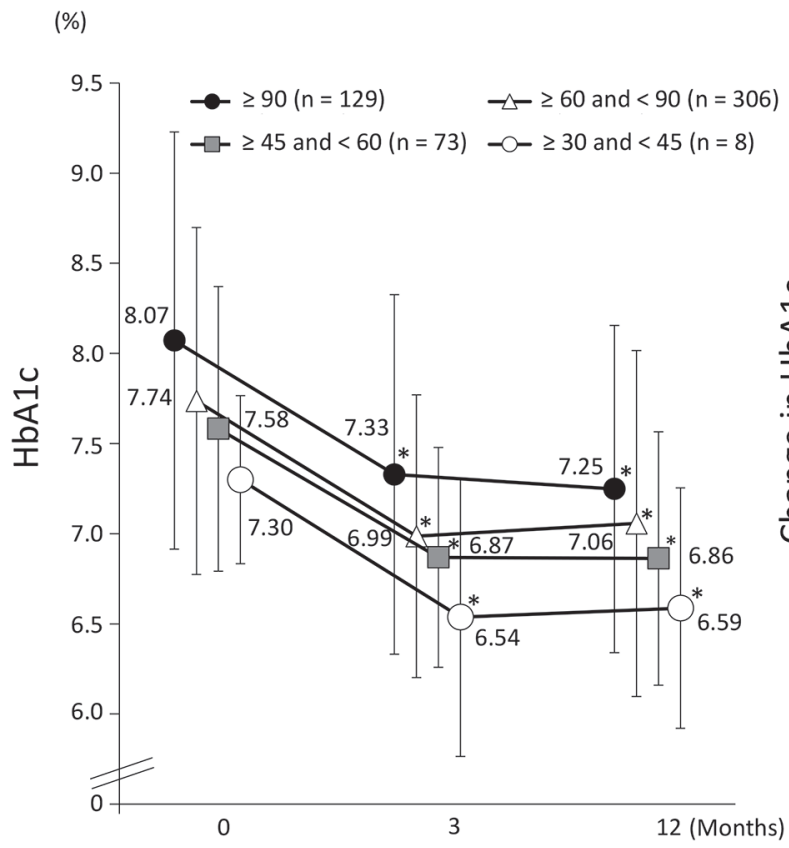

b

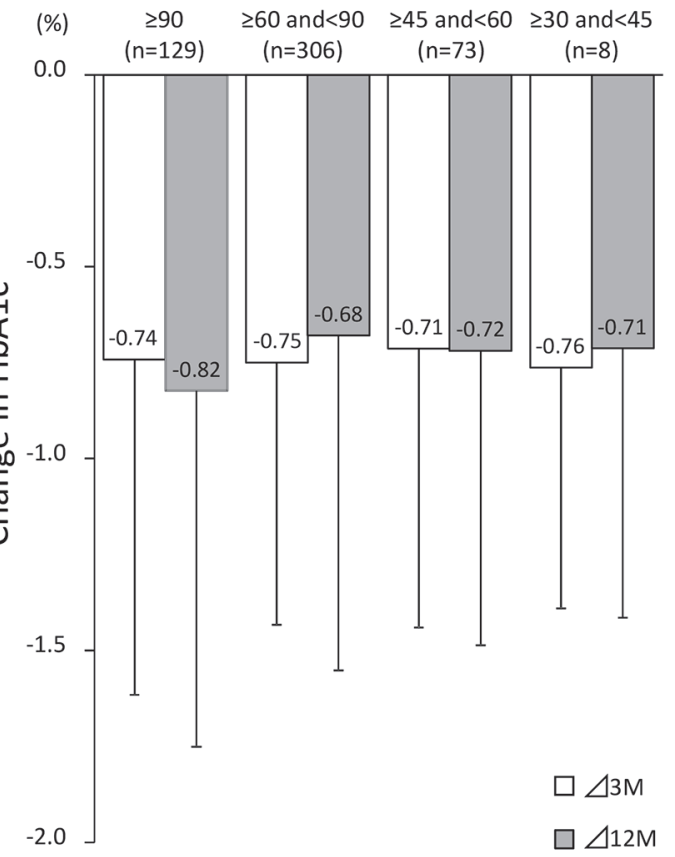

Figure 4. Changes in $\mathrm{HbA1c}$ (categorized by baseline eGFR). (a) eGFR: estimated glomerular filtration rate. ${ }^{*} \mathrm{P}<0.05$ vs. baseline, paired $t$-test. (b) Values presented are mean \pm SD. N.S. ANOVA.

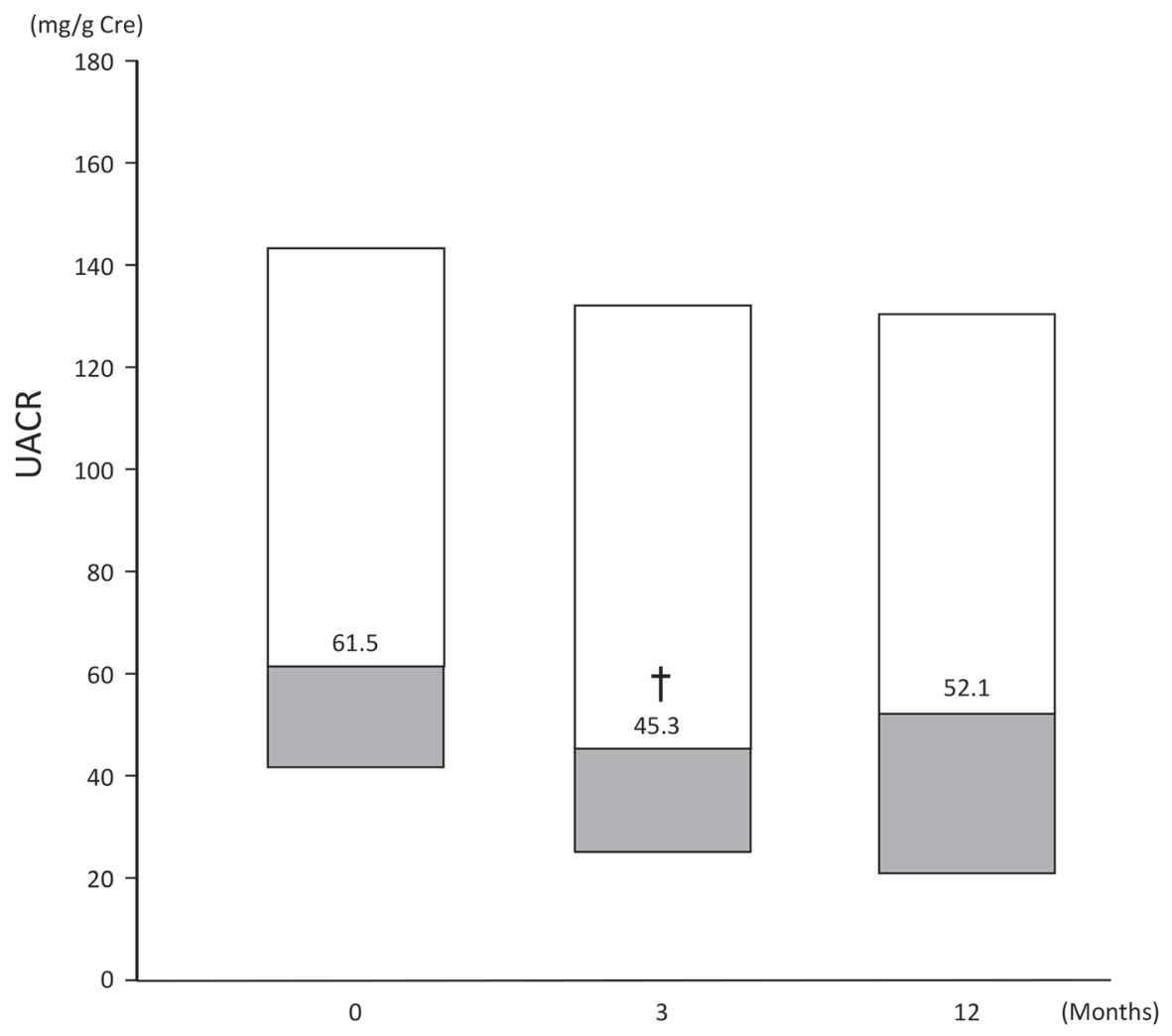

Figure 5. Change in UACR of patients $(n=53)$ with a baseline UACR $\geq 30 \mathrm{mg} / \mathrm{g}$ Cre. Data are first, second, third quartile. Change at: 3 months: $-21.6(-46.8,7.8) ; 12$ months: $-17.1(-39.8,23.8) . \dagger P<0.05$ vs. baseline, Wilcoxon signed rank test. 
Table 2. Single and Multiple Regression Analyses of Change in eGFR From Baseline to 3 Months

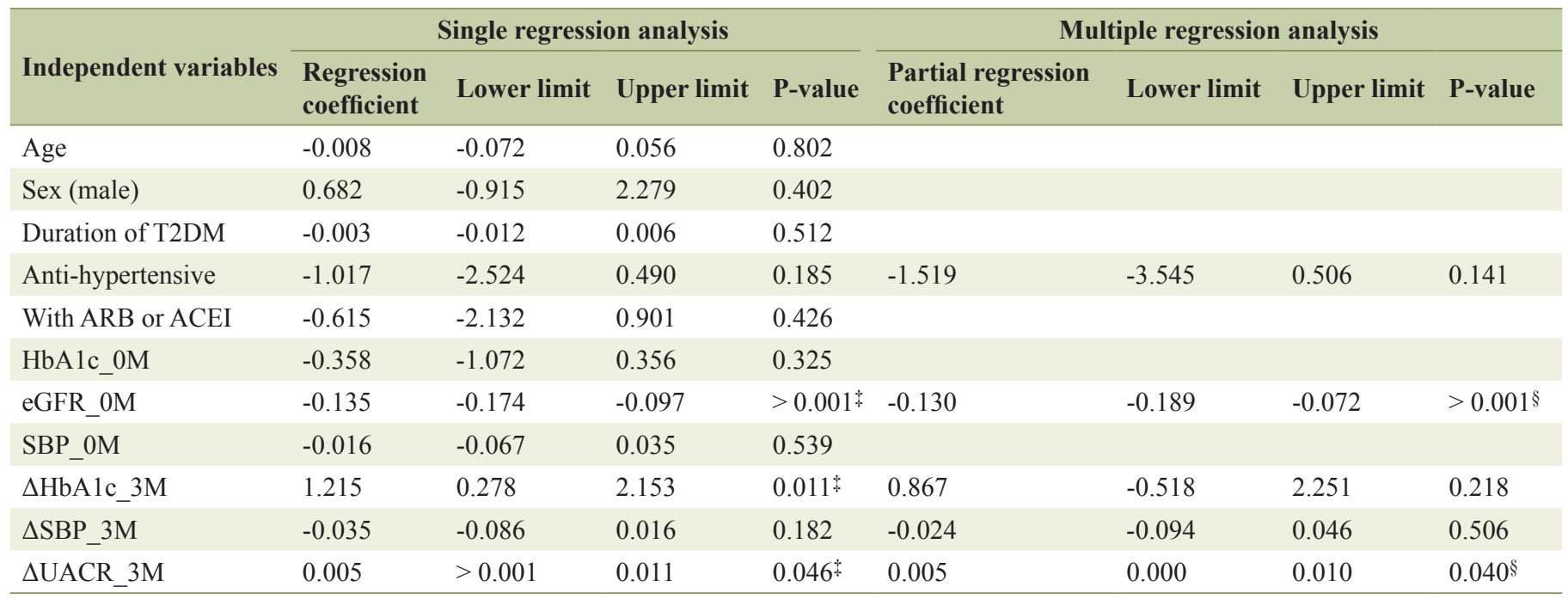

T2DM: type 2 diabetes mellitus; ARB: angiotensin II receptor blocker; ACEl: angiotensin converting enzyme inhibitor; HbA1c: glycated hemoglobin; M: month; eGFR: estimated glomerular filtration rate; $\Delta$ : change in; UACR: urinary albumin-to-creatinine ratio. ${ }^{\ddagger} \mathrm{P}<0.05$ single regression analysis. $\S \mathrm{P}<0.05$ multiple regression analysis.

Enhanced Na excretion by the inhibition of NHE3 activity was also demonstrated in a basic research study on GLP-1 agonists [22]. As in human subjects, administration of GLP-1 was reported to increase $\mathrm{Na}$ excretion and lower glomerular filtration rate in obese men [23]. Correction of excessive filtration by sitagliptin-induced enhancement of $\mathrm{Na}$ excretion could be an underlying factor in the decline in eGFR in this study.

Although the follow-up period was only 1 year in this study, results of long-term administration have been reported in other studies. According to Maeda et al, serum creatinine levels were significantly increased after 1 month of sitagliptin therapy, creatinine levels remained high until after 24 months, and were significantly different from baseline values. Serum creatinine levels showed no further significant increase after 6 months and remained on a plateau until after 24 months [17]. The results of the TECOS study showed a change in eGFR of $-4.0 \pm 18.4 \mathrm{~mL} / \mathrm{min} / 1.73 \mathrm{~m}^{2}$ at 4 years, indicating that prolonged administration does not cause an excessive decrease in glomerular filtration rates.

Currently, a number of DPP-4 inhibitors are available. As most of these are renally excreted, the safety of linagliptin administration [24], which is excreted via the biliary pathway, is attracting attention in the context of patients with reduced renal function, such as elderly patients. However, the results of the present sub-analysis of the JAMP study showed that the use of sitagliptin was not associated with deterioration of renal function in the patients with eGFRs $\geq 30 \mathrm{~mL} / \mathrm{min} / 1.73$ $\mathrm{m}^{2}$. Studies in patients with renal failure also demonstrated the efficacy and safety of sitagliptin administration $[14,25]$. Nevertheless, because no study has directly compared the effects of anti-diabetic drugs on the development and progression of diabetic nephropathy, prevention of the development and progression of diabetic nephropathy requires initiation of strict blood glucose control at an early stage, regardless of the type of hypoglycemic drug used [26-28].
In this study, subjects were divided into four groups according to baseline eGFR. These groups were compared regarding changes in HbA1c levels. In all the groups, a significant decrease in HbA1c levels from baseline was observed after 3 months of treatment; however, no significant betweengroup difference in change in HbA1c levels was observed. This suggests that sitagliptin administration, which can improve the status of blood glucose control regardless of eGFR, provides a useful option for inhibiting the development and progression of diabetic nephropathy.

\section{Study limitations}

The JAMP study was a single-arm study, so we could not compare the subject with the control group. The study was initiated before the approval of sitagliptin therapy for patients with decreased renal function; thus, patients with moderate renal dysfunction were not included in the present study. Therefore, the effect of sitagliptin administration on renal indices in patients with moderate to severe renal dysfunction cannot be discussed based on the results of this study. In addition, UACR was not examined in all patients. Furthermore, change of concomitant drugs might have affected the results after 3 months of treatment; therefore, the results obtained at 12 months may not reflect an association with sitagliptin alone.

\section{Conclusion}

Although sitagliptin administration was associated with a decrease in eGFR in patients with high eGFR, such a decrease was not excessive in patients with low baseline eGFR. Regardless of the eGFR at baseline, sitagliptin administration improved HbAlc levels in patients in this study. Sitagliptin 
therapy, which corrects osmotic diuresis and excessive filtration due to hyperglycemia and improves HbAlc levels, is a useful treatment option for patients with diabetes, with consideration for its influence on renal function.

\section{Acknowledgments}

We would like to express our sincere gratitude to Mr. Shogo Shishikura (MSD K.K.,) (Teikyo University Graduate School of Public Health, MPH) for scientific advice including references for revising the manuscript. We also would like to thank Nouvelle Place Inc. for conducting the data analyses.

\section{JAMP Study Investigators}

Akiko Sato (Maruyama Internal Medicine Clinic); Akira Miyashita (Miyashita Surgery Clinic); Asako Kokubo (Kokubo Clinic); Atsuro Tsuchiya (Tsuchiya Clinic); Dai Hirohara (Hanazono Clinic); Daiji Kogure (Kogure Clinic); Daijo Kasahara (Kasahara Clinic); Hideki Tanaka (Internal Medicine, Seiwa Clinic); Hideki Tanaka (Internal Medicine, Nishiarai Hospital); Hideo Tezuka (Tezuka Clinic); Hiroyuki Kuroki (Internal Medicine, Johsai Hospital); Jun Ogino (Department of Diabetes, Endocrine and Metabolic Diseases, Tokyo Women's Medical University Yachiyo Medical Center); Kanu Kin (Internal Medicine, Nishiarai Lifestyle-Related Diseases Clinic); Kanu Kin (Internal Medicine, Nishiarai Hospital); Kazuko Muto (Tokyo Women's Medical University); Kazuo Suzuki (Kenkokan Suzuki Clinic of Internal Medicine); Keiko Iseki (Iseki Clinic); Keita Watanabe (Watanabe Clinic); Kenshi Higami (Higami Hospital); Kenzo Matsumura (Matsumura Gastroenterological Clinic); Kiyotaka Nakajima (Ebisu Clinic); Koki Shin (Shin Clinic); Kuniya Koizumi (Kuniya Clinic); Maki Saneshige (Mugishima Medical Clinic); Makio Sekine (Sekine Clinic); Makoto Yaida (Urban Heights Clinic); Mari Kiuchi (Physician, Kanauchi Medical Clinic); Mari Mugishima (Mugishima Medical Clinic); Mari Osawa (Department of Diabetes Mellitus, Institute of Geriatrics, Tokyo Women's Medical University); Masae Banno (Banno Medical Clinic); Masahiro Yamamoto (Internal Medicine 1, Shimane University Faculty of Medicine); Masatake Hiratsuka (Higashishinagawa Clinic); Masumi Hosoya (Yasui Clinic); Michika Atsuta (Internal Medicine, Nishiarai Lifestyle-Related Diseases Clinic); Mitsutoshi Kato (Kato Clinic of Internal Medicine); Miwa Morita (Internal Medicine 1, Shimane University Faculty of Medicine); Munehiro Miyamae (Johsai Hospital); Mutsumi Iijima (Abe Hospital); Naomi Okuyama (Shinjuku Mitsui Building Clinic); Nobuo Hisano (Mejiro Medical Clinic); Norihiro Tsuchiya (Omotesando Naika Ganka); Rie Wada (Kanauchi Medical Clinic); Rie Wada (Nerimasakuradai Clinic); Ryuji Momose (Momo Medical Clinic); Sachiko Otake (Tokyo Women's Medical University); Satoko Maruyama (Shinjuku Mitsui Building Clinic); Satoru Takada (Internal Medicine, Social Welfare Corporation Shineikai Takinogawa Hospital); Shigeki Dan (Ube Internal Medicine and Pediatrics Hospital); Shigeki Nishizawa (Nishizawa Medical
Clinic); Shigeo Yamashita (Department of Diabetes and Endocrinology, JR Tokyo General Hospital); Shingo Saneshige (Internal Medicine, Kamiochiai Shin Clinic); Shinichi Teno (Teno Clinic); Shinji Tsuruta (Diabetic Medicine, Itabashi Chuo Medical Center); Shinobu Kumakura (Kumakura Medical Clinic); Sumiko Kijima (Abe Hospital); Takashi Kondo (Kondo Clinic); Takeo Onishi (Internal Medicine, Onishi Clinic); Taku Kudo (Internal Medicine, Social Welfare Corporation Shineikai Takinogawa Hospital); Tatsushi Sugiura (Internal Medicine, Seiwa Clinic); Toshihiko Ishiguro (Kaname Clinic); Yasue Suzuki (Suzuki Medical Clinic); Yasuhiro Tomita (Nakanobu Clinic); Yasuko Takano (Department of Diabetes, Shiseikai Daini Hospital); Yoshihisa Akimoto (Akimoto Yoshi Medical Clinic); Yoshiko Odanaka (Ito Internal Medicine Pediatrics Clinic); Yoshimasa Tasaka (Tokyo Women's Medical University); Yoshitaka Aiso (Internal Medicine, Diabetes, Aiso Clinic); Yukiko Inoue (Inoue Medical Clinic); Yukinobu Kobayashi (Kobayashi Clinic).

\section{Conflict of Interest}

Hiroshi Sakura received honoraria from Mitsubishi Tanabe Pharma Corporation and research grant from Ono Pharmaceutical Co., Ltd. Other authors declare that they have no conflict of interest.

\section{Funding}

The study was funded by the Japan Diabetes Foundation and conducted with the approval of the ethics committee of the Tokyo Women's Medical University (UMIN000019154).

\section{References}

1. Drucker DJ. Enhancing incretin action for the treatment of type 2 diabetes. Diabetes Care. 2003;26(10):29292940.

2. Herman GA, Bergman A, Stevens C, Kotey P, Yi B, Zhao $\mathrm{P}$, Dietrich B, et al. Effect of single oral doses of sitagliptin, a dipeptidyl peptidase-4 inhibitor, on incretin and plasma glucose levels after an oral glucose tolerance test in patients with type 2 diabetes. J Clin Endocrinol Metab. 2006;91(11):4612-4619.

3. Iwamoto Y, Taniguchi T, Nonaka K, Okamoto T, Okuyama K, Arjona Ferreira JC, Amatruda J. Dose-ranging efficacy of sitagliptin, a dipeptidyl peptidase-4 inhibitor, in Japanese patients with type 2 diabetes mellitus. Endocr J. 2010;57(5):383-394.

4. Kashiwagi A, Kadowaki T, Tajima N, Nonaka K, Taniguchi T, Nishii M, Ferreira JC, et al. Sitagliptin added to treatment with ongoing pioglitazone for up to 52 weeks improves glycemic control in Japanese patients with type 2 diabetes. J Diabetes Investig. 2011;2(5):381-390.

5. Kadowaki T, Tajima N, Odawara M, Nishii M, Taniguchi T, Ferreira JC. Addition of sitagliptin to ongoing 
metformin monotherapy improves glycemic control in Japanese patients with type 2 diabetes over 52 weeks. J Diabetes Investig. 2013;4(2):174-181.

6. Tajima N, Kadowaki T, Okamoto T, Sato A, Okuyama K, Minamide T, Arjona Ferreira JC. Sitagliptin added to voglibose monotherapy improves glycemic control in patients with type 2 diabetes. J Diabetes Investig. 2013;4(6):595-604.

7. Harashima SI, Ogura M, Tanaka D, Fukushima T, Wang Y, Koizumi T, Aono M, et al. Sitagliptin add-on to low dosage sulphonylureas: efficacy and safety of combination therapy on glycaemic control and insulin secretion capacity in type 2 diabetes. Int J Clin Pract. 2012;66(5):465476.

8. Bergman AJ, Cote J, Yi B, Marbury T, Swan SK, Smith $\mathrm{W}$, Gottesdiener K, et al. Effect of renal insufficiency on the pharmacokinetics of sitagliptin, a dipeptidyl peptidase-4 inhibitor. Diabetes Care. 2007;30(7):1862-1864.

9. Eligar VS, Bain SC. A review of sitagliptin with special emphasis on its use in moderate to severe renal impairment. Drug Des Devel Ther. 2013;7:893-903.

10. Meyers JL, Candrilli SD, Kovacs B. Type 2 diabetes mellitus and renal impairment in a large outpatient electronic medical records database: rates of diagnosis and antihyperglycemic medication dose adjustment. Postgrad Med. 2011;123(3):133-143.

11. Masakane I, Nakai S, Ogata S, Kimata N, Hanafusa N, Hamano T, Wakai K, et al. An overview of regular dialysis treatment in Japan (As of 31 December 2013). Ther Apher Dial. 2015;19(6):540-574.

12. Kawasaki I, Hiura Y, Tamai A, Yoshida Y, Yakusiji Y, Ikuno Y, Okada M, et al. Sitagliptin reduces the urine albumin-to-creatinine ratio in type 2 diabetes through decreasing both blood pressure and estimated glomerular filtration rate. J Diabetes. 2015;7(1):41-46.

13. Hsieh CJ, Shen FC. The durability of sitagliptin in elderly patients with type 2 diabetes. Clin Interv Aging. 2014;9:1905-1911.

14. Chan JC, Scott R, Arjona Ferreira JC, Sheng D, Gonzalez E, Davies MJ, Stein PP, et al. Safety and efficacy of sitagliptin in patients with type 2 diabetes and chronic renal insufficiency. Diabetes Obes Metab. 2008;10(7):545-555.

15. Mori H, Okada Y, Arao T, Tanaka Y. Sitagliptin improves albuminuria in patients with type 2 diabetes mellitus. J Diabetes Investig. 2014;5(3):313-319.

16. Green JB, Bethel MA, Armstrong PW, Buse JB, Engel SS, Garg J, Josse R, et al. Effect of sitagliptin on cardiovascular outcomes in type 2 diabetes. $\mathrm{N}$ Engl J Med. 2015;373(3):232-242.

17. Maeda H, Kubota A, Kanamori A, Tanaka Y, Terauchi Y, Matsuba I. Effects of sitagliptin on the serum creatinine in Japanese type 2 diabetes. Diabetes Res Clin Pract. 2015;108(3):e42-45.
18. Sakura H, Hashimoto N, Sasamoto K, Ohashi H, Hasumi S, Ujihara N, Kasahara T, et al. Effect of sitagliptin on blood glucose control in patients with type 2 diabetes mellitus who are treatment naive or poorly responsive to existing antidiabetic drugs: the JAMP study. BMC Endocr Disord. 2016;16(1):70.

19. The Japan Diabetes Society. Evidence-based practice guideline for the treatment for diabetes in Japan. 2013;2130 .

20. Takihata M, Nakamura A, Tajima K, Inazumi T, Komatsu Y, Tamura H, Yamazaki S, et al. Comparative study of sitagliptin with pioglitazone in Japanese type 2 diabetic patients: the COMPASS randomized controlled trial. Diabetes Obes Metab. 2013;15(5):455-462.

21. Pacheco BP, Crajoinas RO, Couto GK, Davel AP, Lessa LM, Rossoni LV, Girardi AC. Dipeptidyl peptidase IV inhibition attenuates blood pressure rising in young spontaneously hypertensive rats. J Hypertens. 2011;29(3):520528.

22. Carraro-Lacroix LR, Malnic G, Girardi AC. Regulation of $\mathrm{Na}+\mathrm{H}+$ exchanger NHE3 by glucagon-like peptide 1 receptor agonist exendin-4 in renal proximal tubule cells. Am J Physiol Renal Physiol. 2009;297(6):F1647-1655.

23. Gutzwiller JP, Tschopp S, Bock A, Zehnder CE, Huber AR, Kreyenbuehl M, Gutmann H, et al. Glucagon-like peptide 1 induces natriuresis in healthy subjects and in insulin-resistant obese men. J Clin Endocrinol Metab. 2004;89(6):3055-3061.

24. Barnett AH, Huisman H, Jones R, von Eynatten M, Patel S, Woerle HJ. Linagliptin for patients aged 70 years or older with type 2 diabetes inadequately controlled with common antidiabetes treatments: a randomised, double-blind, placebo-controlled trial. Lancet. 2013;382(9902):14131423.

25. Arjona Ferreira JC, Marre M, Barzilai N, Guo H, Golm GT, Sisk CM, Kaufman KD, et al. Efficacy and safety of sitagliptin versus glipizide in patients with type 2 diabetes and moderate-to-severe chronic renal insufficiency. Diabetes Care. 2013;36(5):1067-1073.

26. Nathan DM, Genuth S, Lachin J, Cleary P, Crofford O, Davis M, Rand L, et al. The effect of intensive treatment of diabetes on the development and progression of longterm complications in insulin-dependent diabetes mellitus. N Engl J Med. 1993;329(14):977-986.

27. Ohkubo Y, Kishikawa H, Araki E, Miyata T, Isami S, Motoyoshi S, Kojima Y, et al. Intensive insulin therapy prevents the progression of diabetic microvascular complications in Japanese patients with non-insulin-dependent diabetes mellitus: a randomized prospective 6-year study. Diabetes Res Clin Pract. 1995;28(2):103-117.

28. Japanese Society of Nephrology. Evidence-based clinical practice guideline for CKD 2013. Clin Exp Nephrol. 2014;18(3):346-423. 